\title{
openheart Higher number of live births is associated with left ventricular diastolic dysfunction and adverse cardiac remodelling among US Hispanic/ Latina women: results from the Echocardiographic Study of Latinos
}

\author{
Shivani R Aggarwal, ${ }^{1}$ David M Herrington, ${ }^{1}$ Catherine J Vladutiu, ${ }^{2}$ Jill C Newman, ${ }^{1}$ \\ Katrina Swett, ${ }^{1}$ Franklyn Gonzalez, ${ }^{2}$ Jorge R Kizer, ${ }^{3}$ Michelle A Kominiarek, ${ }^{4}$ \\ Karen M Tabb, ${ }^{5}$ Linda C Gallo, ${ }^{6}$ Gregory A Talavera, ${ }^{6}$ Barry E Hurwitz, ${ }^{7}$ \\ Carlos J Rodriguez ${ }^{1}$
}

To cite: Aggarwal SR, Herrington DM, Vladutiu CJ, et al. Higher number of live births is associated with left ventricular diastolic dysfunction and adverse cardiac remodelling among US Hispanic/Latina women: results from the Echocardiographic Study of Latinos. Open Heart 2017;4:e000530. doi:10.1136/ openhrt-2016-000530

Received 2 September 2016 Revised 6 September 2016 Accepted 17 November 2016

\section{(a) CrossMark}

${ }^{1}$ Wake Forest School of Medicine, Winston-Salem, North Carolina, USA

${ }^{2}$ University of North Carolina, Chapel Hill, North Carolina, USA ${ }^{3}$ Albert Einstein College of Medicine, Bronx, New York, USA ${ }^{4}$ University of Illinois at Chicago, Chicago, Illinois, USA

${ }^{5}$ University of Illinois UrbanaChampaign, Urbana, Illinois, USA ${ }^{6}$ San Diego State University, San Diego, California, USA ${ }^{7}$ University of Miami, Miami, Florida, USA

Correspondence to Dr Shivani R Aggarwal; saggarwa@wakehealth.edu

\section{ABSTRACT}

Introduction Female sex is a risk factor for heart failure with preserved ejection fraction (HFpEF). Previous literature suggests that some diastolic dysfunction (DD) develops during pregnancy and may persist postdelivery. Our objective was to examine the relationship between parity and cardiac structure and function in a populationbased cohort.

Methods Participants included 1172 Hispanic/Latina women, aged $\geq 45$ years, enrolled in the Echocardiographic Study of Latinos from four US communities (Bronx, Miami, San Diego and Chicago). Standard echocardiographic techniques were used to measure cardiac volumes, left ventricular mass, systolic and diastolic function. Using sampling weights and survey statistics, multivariable linear and logistic regression models were constructed adjusting for age, body mass index, diabetes or prediabetes, systolic blood pressure, use of antihypertensive medications, smoking, total cholesterol and high-density lipoprotein cholesterol.

Results In the target population, $5.0 \%$ were nulliparous (no live births) and $10.5 \%$ were grand multiparous ( $\geq 5$ live births). Among the nulliparous women, $46 \%$ had DD as compared with $51 \%-58 \%$ of women with $1-4$ live births and $81 \%$ of women with $\geq 5$ live births $(p<0.01)$. In full multivariate models, higher parity was significantly associated with greater left ventricular end-systolic volumes, end-diastolic volumes, left atrial volume indices and presence of DD (all $p<0.01$ ) but was not associated with ejection fraction. The log odds for having any grade of $\mathrm{DD}$ in grand-multiparous women was over three times that seen in nulliparous women $(\mathrm{OR}=3.4,95 \% \mathrm{Cl} 1.5$ to $7.9, p<0.01$ ) in models further adjusted for income and education.

Conclusions Higher parity is associated with increased cardiac mass, volumes and the presence of DD. Further studies are needed to elucidate this apparent deleterious relation and whether parity can help explain the increased risk of $\mathrm{HFpEF}$ in women.

\section{KEY MESSAGES}

What is already known about this subject?

- Literature indicates that having six or more pregnancies is associated with increased risk of cardiovascular disease and possibly heart failure. Mortality is also higher in women with very high parity. However the underlying pathophysiology for these associations is unclear. The Multi-Ethnic Study of Atherosclerosis showed an independent positive association between parity and left ventricular mass (LVM), LV end-systolic volume and an independent inverse association between parity and LV ejection fraction. However there is no prior literature on the association of diastolic dysfunction (DD) with parity.

What does this study add?

- This study shows a strong association between high parity (five or more live births) and DD which was previously unknown. It also provides more evidence for increasing LVM, and end-systolic and end-diastolic volumes previously noted.

How might this impact on clinical practice?

- This study indicates there may be a new, novel risk factor for DD and this may translate to a risk factor for heart failure with preserved ejection fraction. If further studies confirm this finding, these women with very high parity may need additional monitoring or treatment. Further, preventive strategies will need to be explored.

\section{INTRODUCTION}

Heart failure with preserved ejection fraction (HFpEF) is a growing public health problem with recent data revealing that the prevalence of HFpEF is increasing while its mortality 
rate remains unchanged. ${ }^{1}$ Across multiple epidemiological studies women are at a significantly higher risk for HFpEF than men without any clear explanation for this disparity. ${ }^{2-4}$ The process of childbearing and childbirth, during which the cardiovascular system undergoes immense adaptations, may be a potential explanation for these differences. During pregnancy the maternal cardiovascular system undergoes extensive cardiovascular adaptations including an increase in cardiac mass by $30 \%-50 \%$ as well as increased cardiac output, stroke volume and heart rate. ${ }^{5-9}$ Though complete reversal is thought to occur following delivery, several studies have suggested persistence of some structural changes.

Literature from as early as the mid-1980s to 1990s indicates that having six or more pregnancies is associated with increased risk of cardiovascular disease (CVD) and possibly heart failure (HF) ${ }^{10-13}$ and that this association persists after adjustment for pregnancy complications. ${ }^{14}$ There have been few studies to explain the underlying pathophysiology for this possible association between multiple pregnancies and CVD or HF although a recent study did show an association between parity and some components of the metabolic syndrome. ${ }^{15}$ A small study suggested that diastolic dysfunction (DD) and left ventricular hypertrophy (LVH) persisted for 2 months in the peripartum period while systolic function normalised within a week. ${ }^{16} \mathrm{~A}$ study in primiparous (one live birth) and multiparous (>one live birth) women, both during pregnancy and up to 1 year post-delivery, showed that the cardiovascular adaptations to the initial pregnancy begin early, persist postpartum even 1 year after delivery and are enhanced by subsequent pregnancies. ${ }^{17}$ The Multi-Ethnic Study of Atherosclerosis showed an independent positive association between parity and LV mass (LVM), LV end-systolic volume (ESV) and an independent inverse association between parity and LV ejection fraction (LVEF). ${ }^{18}$ However there is no prior literature on the association of DD with parity. Our objective was to examine the relationship between parity and various echocardiographic parameters including LV volumes, LVM, LVEF, left atrial volume indices (LAVI) and DD in a population-based cohort. We hypothesised that higher parity would be associated with abnormalities of cardiac structure and function, in particular, the presence of DD.

\section{METHODS}

The Hispanic Community Health Study /Study of Latinos (HCHS/SOL) is a community-based cohort study of self-identified Hispanic/Latino individuals from randomly selected households in four US sites (Chicago, Illinois; Miami, Florida; Bronx, New York; San Diego, California). The baseline examination was conducted from 2008 to 2011. The HCHS/SOL sample design and cohort selection has been previously described. ${ }^{19}$ The Echocardiographic Study of Latinos (ECHO-SOL), an ancillary study to the HCHS/SOL main study, was designed to provide echocardiographic parameters characterising cardiac structure and function in a representative baseline subsample of HCHS/SOL participants 45 years and older. A detailed description of the design, rationale and methods has been described elsewhere. ${ }^{20}$ Briefly, 1818 participants were enrolled in ECHO-SOL from October 2011 through June 2014 using a stratified random sampling design to ensure a balanced number of participants representative of the overall HCHS-SOL population 45 years of age or older were enrolled at each centre. ${ }^{21}$ Echocardiographic studies were sent electronically through a fully encrypted, regulatory compliant secure server to the ECHO-SOL reading centre at Wake Forest School of Medicine.

For this analysis only Hispanic/Latina women (1172 in total) who were enrolled in ECHO-SOL are included.

\section{Echocardiographic outcome measurements}

To maintain consistency across sites, one ultrasound imaging platform was used: Philips Ultrasound IE-33 or Sonos 5500/7500 with software V.D.2 or higher interfaced with a standard $2.5 \mathrm{MHz}$ to $3.5 \mathrm{MHz}$ phased-array probe. Experienced sonographers at each field imaging centre performed standard echocardiographic examinations, including M-mode, two-dimensional, spectral, colour flow and tissue Doppler studies according to the recommendations of the American Society of Echocardiography (ASE). ${ }^{22} 23$

LV chamber size and wall thickness were assessed by multiple linear dimensions that were measured from the parasternal long-axis view for determining LVM and have been well validated in autopsy studies. ${ }^{24} 25$ Using volumetric assessments from the apical fourchamber and two-chamber views, LV end-diastolic volume (EDV) and ESV were derived using the biplane method of discs. ${ }^{26}$ LVEF was calculated from EDV and ESV estimates, using the following formula: LVEF = (EDV - ESV)EDV.

For echocardiographic assessment of DD, Doppler recording of transmitral inflow velocities (mitral $\mathrm{E}$ and A waves) was performed in the apical four-chamber view with the pulse-wave Doppler sample volume placed in the mitral valve orifice at the level of the leaflet tips during mid-diastole. Mitral inflow velocity measurements were made from the cycles which exhibited the narrowest spectral dispersion and the highest peak velocity in early diastole. ${ }^{22}$ Tissue Doppler imaging was used to evaluate early mitral diastolic $\left(\mathrm{e}^{\prime}\right)$ annular velocities from the apical four-chamber view, and the relationship of the amplitude of $\mathrm{E} / \mathrm{e}^{\prime}$ ratio was examined per recommendation of the most recent ASE and European Association of Echocardiography guidelines, averaging septal and lateral values. ${ }^{27} \mathrm{LA}$ volumes were determined from apical two-chamber and four-chamber views and indexed to body surface area. DD was graded following an algorithm that combined ASE guidelines ${ }^{28}$ and Redfield criteria ${ }^{29}$ using three echocardiographic parameters: E/A ratio, $\mathrm{E} / \mathrm{e}^{\prime}$ ratio and LAVI as previously described and shown in figure $1 .^{30}$ 


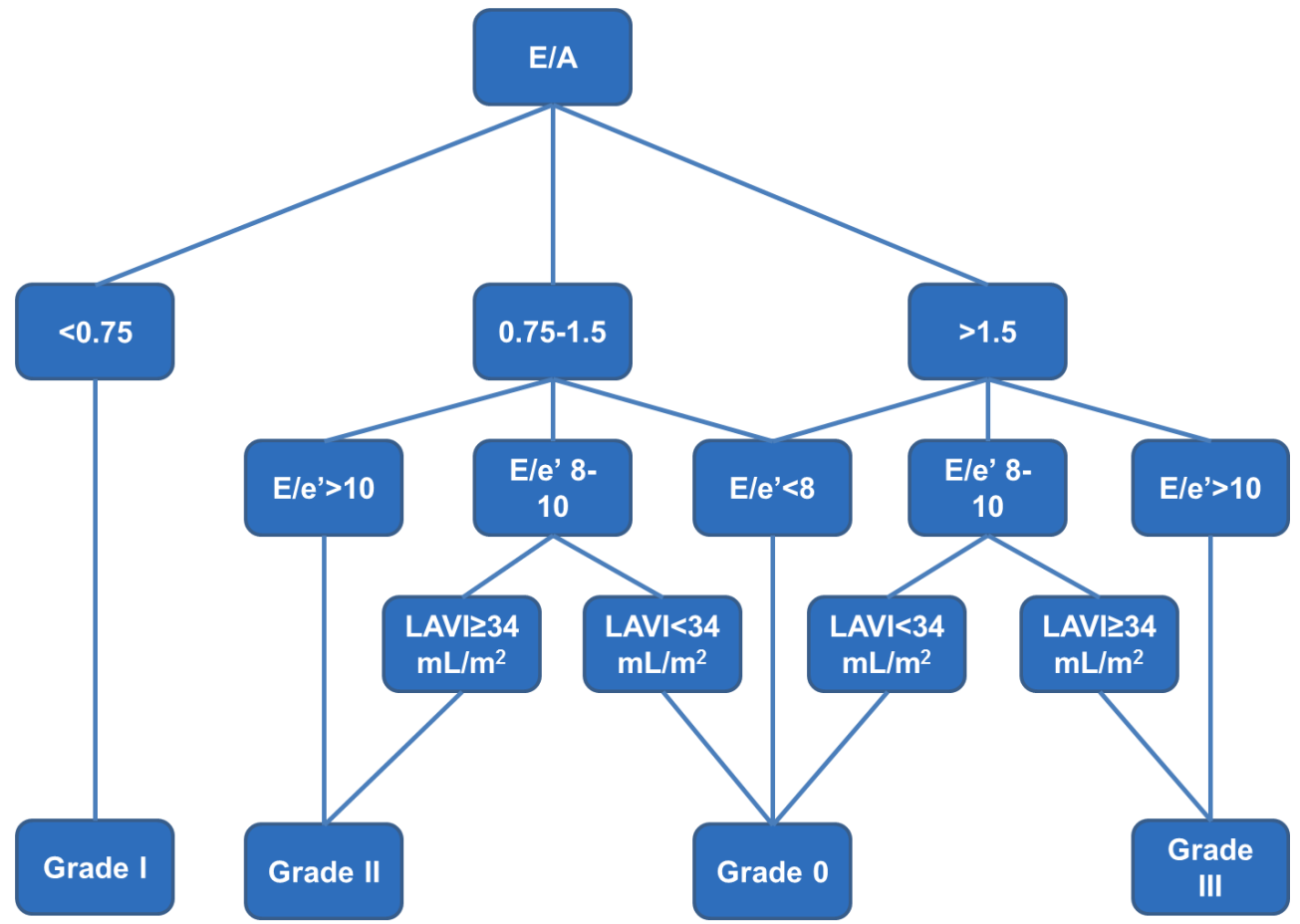

Figure 1 Method of assessment of grade of diastolic dysfunction in ECHO-SOL.30 ECHO-SOL, Echocardiographic Study of Latinos; LAVI, left atrial volume index.

We also assessed DD using the new ASE/European Association of Cardiovascular Imaging (EACVI) guidelines published in April $2016^{31}$ for the purpose of performing a sensitivity analysis to assess whether this would significantly alter our results. In this algorithm E/A ratio of $<0.8$ was taken as grade I, $0.8-2$ as normal or grade II and $>2$ as grade III. In addition for the presence of grade III DD 2 additional criteria were needed: LAVI $\geq 34 \mathrm{~mL} /$ $\mathrm{m}^{2}$ and $\mathrm{E} / \mathrm{e}^{\prime} \geq 14$. To differentiate between normal and grade II DD, six criteria were evaluated: $\mathrm{E} / \mathrm{e}^{\prime}$ of $\geq 14$, LAVI $\geq 34 \mathrm{~mL} / \mathrm{m}^{2}$, tricuspid regurgitation velocity $\geq 2.8 \mathrm{~m} / \mathrm{sec}$, pulmonary vein $\mathrm{S} / \mathrm{D}$ ratio $\leq 1$, Ar-A duration of $\geq 30 \mathrm{msec}$ and isovolumic relaxation time $\leq 100 \mathrm{msec}$. Presence of a single criterion was considered normal diastolic function and up to two criteria was considered grade II DD. An indeterminate category was added for those where four of six criteria were unavailable.

All ECHO-SOL echocardiograms were analysed and interpreted centrally at Wake Forest School of Medicine (Winston-Salem, North Carolina), were read by a certified technical reader and over-read by a board-certified cardiologist with expertise in echocardiography (CJR). Over-reads of echocardiograms were performed to confirm the accuracy of key quantitative measurements and to identify clinically important findings. ${ }^{32} 33$ Interreader and intrareader reproducibility was assessed and previously reported. ${ }^{20}$

\section{Independent measures}

Parity was defined as the number of prior live births as reported by women on the interviewer-administered questionnaire. For this analysis, parity was defined two ways: (1) Six-level variable with parity categorised as none (nulliparity), 1, 2, 3, 4 and $\geq 5$ (grand-multiparity) prior live births; (2) Three-level variable with categories $0,1-4$ and $\geq 5$ live births. The first definition was used in linear regression and the latter in ordinal logistic regression modelling.

HCHS/SOL baseline clinical parameters have been previously described. ${ }^{34-36}$ Briefly, trained personnel administered a standardised questionnaire assessing participant sociodemographic characteristics, such as age and sex. Socioeconomic status was assessed using information collected on educational attainment and income. Education was categorised as $<$ high school, high school or equivalent, and $>$ high school and income was classified as $<\$ 20000, \$ 20000-\$ 40000$ and $>\$ 40000$. Self-report questionnaires were used to assess whether participants have ever smoked and/or were current smokers. Trained technicians measured each participant's height and weight twice and then averaged these two measures to calculate body mass index $(\mathrm{BMI}=$ weight $(\mathrm{kg}) / \mathrm{height}$ $\left.\left(\mathrm{m}^{2}\right)\right)$. Medical personnel measured resting systolic blood pressure (SBP) and diastolic blood pressure using a standardised protocol performed at the baseline visit. Seated resting blood pressures were measured in triplicate and the average of the second and third readings were used for analysis. A single blood pressure measurement at the time of the echocardiogram correlated well with these measures. Information on antihypertensive treatment used by participants was obtained via scanning of medication package bar code symbols, transcription of pill bottle labels and survey interviews. The variable used in the 
statistical analysis was dichotomous - whether the participant was on antihypertensive medications or not. Type 2 diabetes was defined using one or more of the following criteria: (1) fasting serum glucose $>126 \mathrm{mg} / \mathrm{dL}$, (2) oral glucose tolerance test $>200 \mathrm{mg} / \mathrm{dL}$, (3) self-reported diabetes, (4) $\mathrm{Hb} \mathrm{A} 1 \mathrm{C}>6.5 \%$ or (5) taking antidiabetic medication or insulin. Prediabetes was defined as $\mathrm{HbAlC}$ $\geq 5.7$ but $<6.5 \%$ in the absence of treatment. Total cholesterol and high-density lipoprotein (HDL) cholesterol were measured on blood samples obtained after an overnight fast.

\section{Statistical analysis}

Descriptive summary statistics were generated overall and by six-level parity for the analytical sample. Weighted means and standard errors were reported for continuous variables and weighted frequencies for categorical variables. All analyses accounted for cluster sampling, the use of stratification in sample selection and sample weights, which were incorporated to accommodate for unequal probabilities of sample selection and to partially adjust for bias due to differential non-response in sample selection at the household and person levels. Calibration to the 2010 Census characteristics by age, sex and Hispanic/ Latino background in each study site target population was used to derive the adjusted weights.

Bivariate associations between baseline characteristics and parity were assessed using survey linear regression models and $\chi^{2}$ tests. Multilevel, multivariable survey logistic regression models assessed the relationship between parity and the presence of abnormal LV geometry and any grade DD (grades I-III). Comparable multivariable sequential survey linear regression models were used to assess the relationship between parity and EDV, ESV, LAVI, LVM and LVEF. The models were adjusted for covariates as follows:

Model 1: Age

Model 2: Age, BMI, SBPs, diabetes or prediabetes and antihypertension medication use

Model 3: Smoking status, total cholesterol and HDL cholesterol in addition to variables in model 2

Model 4: Education and household income in addition to variables in model 3

Further sensitivity analyses were performed by the addition of ethnic subgroup to model 1 and the recruitment site to model 3 to see if this resulted in any alteration in the estimates. Sensitivity analyses were also performed with presence of absence of DD using the new 2016 guidelines to assess for any change in the relationship between parity and DD with the newer method of assessment of DD.

$\beta$ Estimates and standard errors were reported for linear models, as well as ORs and 95\% CIs for categorical measures. For linear outcomes, line graphs were used to visually represent the least square means of the fully adjusted model by increasing levels of parity. Parity levels by percentage of participants with DD were represented using a bar chart. A p value of $<0.05$ was considered statistically significant and all analyses were performed using SAS V.9.4 software (SAS Institute, Cary, North Carolina, USA).

\section{RESULTS}

\section{Subjects}

Among women in the target population, $5.0 \%$ were nulliparous (no live births) and $10.5 \%$ were grand multiparous ( $\geq 5$ live births). Overall this population was high risk for CVD due to their high BMIs and high occurrences of diabetes, hypertension and abnormal cholesterol (table 1). A larger number of births was seen in individuals with higher BMI, lower educational attainment and less income (table 1). Current smoking rates varied by level of parity such that women with four prior live births had the highest proportion of smokers (22.7\%), followed by women with one prior live birth $(21.2 \%)$ and those with no prior births $(17.3 \%)$. Women with up to five live births had a higher SBP and likelihood of diabetes.

\section{Cardiac volumes}

Women within all categories above two or more live births had significantly higher LV EDVs ranging from $6.3 \mathrm{~mL}$ to $8.3 \mathrm{~mL}$ higher in these categories after adjustment for covariates (table 2; figure 2) compared with nulliparous women. Similarly, women with two or more live births had significantly higher LV ESVs compared with nulliparous women with volumes ranging $1.9 \mathrm{~mL}$ to $3.1 \mathrm{~mL}$ higher across parity categories after adjustment for covariates (table 2; figure 2). LAVI was also higher by $\sim 3 \mathrm{~mL} / \mathrm{m}^{2}$ in women with four or more live births compared with nulliparous women.

\section{Left ventricular mass and systolic function}

LVM was significantly higher in women with one, two, four or up to five live births compared with nulliparous women, with mass as much as 20 grams higher in women with four live births after adjustment for covariates (table 2; figure 2). Women with three live births did not have significantly higher mass compared with nulliparous women. Though a decline in LVEF was estimated with higher parity, there was no statistically significant association between parity and LVEF.

\section{Diastolic dysfunction}

The overall prevalence of any grade DD in the target population was $52 \%$. Among the nulliparous women, $46 \%$ had any grade DD as compared with $51 \%-58 \%$ of women with one to four live births and $81 \%$ of women with up to five live births $(\mathrm{p}=0.006)$ (figure 3$)$. In a multivariable logistic regression model, the log odds for having any grade of DD in grand-multiparous women was over three times that of nulliparous women (adjusted $\mathrm{OR}=3.2$, $95 \%$ CI 1.5 to $6.7, \mathrm{p}<0.01$ ), adjusting for age, BMI, diabetes or prediabetes, SBP, use of antihypertensive medications, smoking, total cholesterol and HDL cholesterol (figure 4). This association remained unchanged 
Table 1 Baseline characteristics (frequency or mean (SE)) of female participants in the Echocardiographic Study of Latinos $(n=1172)$

\begin{tabular}{|c|c|c|c|c|c|c|c|c|}
\hline & Overall & $\begin{array}{l}\text { O Births } \\
(5.0 \%)\end{array}$ & $\begin{array}{l}1 \text { Birth } \\
(13.5 \%)\end{array}$ & $\begin{array}{l}2 \text { Births } \\
(32.3 \%)\end{array}$ & $\begin{array}{l}3 \text { Births } \\
(25.3 \%)\end{array}$ & $\begin{array}{l}4 \text { Births } \\
(13.4 \%)\end{array}$ & $\begin{array}{l}\text { 5+ Births } \\
(10.5 \%)\end{array}$ & p Value* \\
\hline Age & $56.1(0.5)$ & $56.1(1.2)$ & $54.0(0.7)$ & $55.4(0.8)$ & $56.2(1.4)$ & $56.2(1.0)$ & $60.7(1.1)$ & $<0.01$ \\
\hline $\mathrm{BMI}\left(\mathrm{kg} / \mathrm{m}^{2}\right)$ & $31.0(0.3)$ & $30.3(0.9)$ & $29.9(0.5)$ & $30.4(0.4)$ & $31.4(1.0)$ & $32.1(0.5)$ & $32.2(0.7)$ & 0.01 \\
\hline Education & & & & & & & & $<0.01$ \\
\hline Less than HS & 33.9 & 23.3 & 21.9 & 20.7 & 36.5 & 54.5 & 62.3 & \\
\hline HS or equivalent & 20.8 & 30.1 & 21.4 & 20.4 & 23.0 & 16.1 & 18.1 & \\
\hline Greater than HS & 45.3 & 46.6 & 56.7 & 58.9 & 40.5 & 29.4 & 19.6 & \\
\hline Income & & & & & & & & 0.01 \\
\hline$<\$ 20,000$ & 57.9 & 52.2 & 58.5 & 59.8 & 49.5 & 53.5 & 80.8 & \\
\hline$\$ 20000$ to $\$ 40000$ & 30.9 & 29.1 & 32.4 & 26.8 & 37.4 & 37.5 & 17.3 & \\
\hline$>\$ 40000$ & 11.2 & 18.3 & 9.1 & 13.5 & 13.1 & 9.0 & 1.9 & \\
\hline Current smoker & 13.9 & 17.3 & 21.2 & 12.7 & 8.5 & 22.7 & 9.0 & $<0.01$ \\
\hline SBP & $134.3(0.9)$ & $130.2(2.5)$ & $130.5(1.9)$ & $132.8(1.2)$ & $135.6(2.5)$ & $136.4(2.5)$ & $140.2(2.0)$ & $<0.01$ \\
\hline Diabetes mellitus & & & & & & & & 0.01 \\
\hline No diabetes & 32.0 & 32.5 & 38.5 & 38.4 & 25.1 & 32.9 & 19.6 & \\
\hline Prediabetes & 40.0 & 51.2 & 37.5 & 37.3 & 45.5 & 39.8 & 33.3 & \\
\hline Diabetes & 28.0 & 16.3 & 24.1 & 24.3 & 29.4 & 27.3 & 47.1 & \\
\hline Anti-HTN meds & 27.6 & 20.2 & 20.9 & 23.1 & 27.8 & 33.8 & 44.7 & 0.05 \\
\hline Total cholesterol (mg/dL) & $214.2(1.7)$ & $223.0(7.8)$ & $216.3(3.9)$ & $214.0(3.1)$ & $216.1(3.0)$ & $215.3(3.7)$ & $202.1(6.1)$ & 0.30 \\
\hline HDL cholesterol (mg/dL) & $53.5(0.5)$ & $54.7(1.7)$ & $52.8(1.1)$ & $53.3(1.1)$ & $54.1(0.8)$ & $54.1(0.9)$ & $51.9(1.5)$ & 0.76 \\
\hline
\end{tabular}

${ }^{*} p$ Value from survey linear regression models for continuous measures and $\chi^{2}$ tests for categorical measures.

BMI, body mass index; HDL, high-density lipoprotein; HS, high school; HTN, hypertension; SBP, systolic blood pressure.

after further adjustment for education and income as proxies for socioeconomic status.

No significant change noted in the $\beta$ estimates or $\mathrm{p}$ values with further adjustment for Hispanic background subgroup or site in separate models for our sensitivity analyses.

Using the newer guideline based assessment of DD in our sensitivity analysis the prevalence of DD was lower with overall prevalence of $43.5 \%$. Among the nulliparous women, $36 \%$ had any grade DD as compared with $42 \%$ of women with one to four live births and $61 \%$ of women with up to five live births $(\mathrm{p}=0.01)$. The overall association remained unchanged.

\section{DISCUSSION}

\section{Summary of findings compared with prior literature}

Among 1172 Hispanic women aged 45 years and older, high parity was associated with increased EDV, ESV, LVM, LAVI and DD. Our findings are in agreement with analyses from the Multi-Ethnic Study of Atherosclerosis $^{18}$ which noted increased EDV, ESV and LVM with increasing parity. In contrast, we did not find a statistically significant association between parity and LVEF. We also noted a significantly higher prevalence of any grade DD in grand-multiparous women compared with nulliparous women with an OR as high as 3.4 after adjustment for covariates. To our knowledge, this is the first study to evaluate the association between DD and parity. Additionally, this finding is consistent with prior studies that have shown a higher incidence of CVD in women with a large number of pregnancies. ${ }^{10-14}$ In our study we have exclusively studied purely the echocardiographic presence of DD. a subclinical parameter, not the presence of clinical HFpEF. Whether our finding of increased echocardiographic DD translates to HFpEF, remains to be studied.

Our study showed a higher prevalence of DD compared with prior European studies such as the Flemish Study on Environment, Genes and Health Outcomes (FLEMENGHO) and European Project on Genes in Hypertension (EPOGH) ${ }^{37}{ }^{38}$ However, comparison of our current study to these prior studies is difficult since those populations were not exclusively in women, did not include any Hispanic/Latina participants and the demographics and risk factor profile in these studies differed significantly from ours with their participants being younger, having lower blood pressure and overall less risk factors such as diabetes and obesity. The community based study which assessed the prevalence of DD in Olmstead County and recruited a similar age group of participants (over 45 years) also had a lower prevalence of DD but again the average BMI was lower and the prevalence of diabetes was also significantly lower. ${ }^{29}$ 
Table 2 Multilevel multivariable linear regression models of association between parity and cardiac volumes and mass

\begin{tabular}{|c|c|c|c|c|c|c|c|c|c|c|c|}
\hline & & \multicolumn{10}{|l|}{ Parity } \\
\hline & & \multicolumn{2}{|l|}{$\geq 5$ vs 0} & \multicolumn{2}{|l|}{4 vs 0} & \multicolumn{2}{|l|}{3 vs 0} & \multicolumn{2}{|l|}{2 vs 0} & \multicolumn{2}{|l|}{1 vs 0} \\
\hline & & $\begin{array}{l}\beta \text { Estimate } \\
(\mathrm{SE})^{\star}\end{array}$ & $p$ Value & $\begin{array}{l}\beta \text { Estimate } \\
\text { (SE)* }\end{array}$ & $p$ Value & $\begin{array}{l}\beta \text { Estimate } \\
(\mathrm{SE})^{\star}\end{array}$ & $p$ Value & $\begin{array}{l}\beta \text { Estimate } \\
(\mathrm{SE})^{\star}\end{array}$ & $p$ Value & $\begin{array}{l}\beta \text { Estimate } \\
(\mathrm{SE})^{*}\end{array}$ & $p$ Value \\
\hline \multirow{4}{*}{$\begin{array}{l}\text { EDV } \\
(\mathrm{mL})\end{array}$} & Model 1 & $8.83(3.2)$ & 0.01 & $8.42(3.1)$ & 0.01 & $8.45(3.4)$ & 0.01 & 7.57 (2.7) & 0.01 & $2.08(2.8)$ & 0.45 \\
\hline & Model 2 & $7.00(2.6)$ & 0.01 & $6.31(2.5)$ & 0.01 & $7.17(2.4)$ & 0.00 & $7.11(2.2)$ & 0.00 & $2.47(2.4)$ & 0.30 \\
\hline & Model 3 & $7.11(2.6)$ & 0.01 & $6.02(2.4)$ & 0.01 & $7.34(2.3)$ & 0.00 & $7.24(2.2)$ & 0.00 & $2.37(2.3)$ & 0.30 \\
\hline & Model 4 & $8.27(2.9)$ & 0.00 & $6.29(2.5)$ & 0.01 & $7.28(2.3)$ & 0.00 & $8.17(2.7)$ & 0.00 & $2.06(2.5)$ & 0.41 \\
\hline \multirow{4}{*}{$\begin{array}{l}\text { ESV } \\
\text { (mL) }\end{array}$} & Model 1 & $4.30(1.4)$ & 0.00 & $3.46(1.2)$ & 0.01 & $3.27(1.3)$ & 0.01 & 3.28 (1.3) & 0.01 & $1.86(1.2)$ & 0.11 \\
\hline & Model 2 & $3.12(1.3)$ & 0.02 & $2.27(1.1)$ & 0.03 & $2.41(1.0)$ & 0.02 & $2.78(1.2)$ & 0.02 & $1.75(1.1)$ & 0.11 \\
\hline & Model 3 & $3.12(1.3)$ & 0.02 & $2.09(1.0)$ & 0.04 & $2.52(1.0)$ & 0.01 & $2.84(1.2)$ & 0.01 & $1.66(1.1)$ & 0.12 \\
\hline & Model 4 & $3.12(1.4)$ & 0.03 & $1.94(1.1)$ & 0.07 & $2.34(1.0)$ & 0.02 & $2.96(1.3)$ & 0.03 & $1.36(1.2)$ & 0.25 \\
\hline \multirow{4}{*}{$\begin{array}{l}\text { LAVI } \\
\left(\mathrm{mL} / \mathrm{m}^{2}\right)\end{array}$} & Model 1 & $3.19(1.3)$ & 0.02 & $3.50(1.3)$ & 0.01 & $2.00(1.2)$ & 0.09 & $1.58(1.2)$ & 0.17 & $1.28(1.3)$ & 0.32 \\
\hline & Model 2 & $2.68(1.3)$ & 0.05 & $3.26(1.3)$ & 0.01 & $1.75(1.2)$ & 0.15 & $1.45(1.2)$ & 0.22 & $1.31(1.3)$ & 0.31 \\
\hline & Model 3 & $2.70(1.3)$ & 0.04 & $3.21(1.3)$ & 0.02 & $1.81(1.2)$ & 0.13 & $1.51(1.2)$ & 0.20 & $1.32(1.3)$ & 0.31 \\
\hline & Model 4 & $2.69(1.4)$ & 0.06 & $3.10(1.4)$ & 0.03 & $1.81(1.2)$ & 0.14 & $1.78(1.2)$ & 0.15 & $1.09(1.3)$ & 0.41 \\
\hline \multirow[t]{4}{*}{ LVM (gm) } & Model 1 & $24.85(7.9)$ & 0.00 & $26.68(6.0)$ & 0.00 & $7.53(4.8)$ & 0.11 & $11.30(4.5)$ & 0.01 & $9.54(5.3)$ & 0.07 \\
\hline & Model 2 & 17.04 (6.5) & 0.01 & $19.60(4.9)$ & 0.00 & $2.63(5.5)$ & 0.63 & $9.74(3.8)$ & 0.01 & $9.54(4.4)$ & 0.03 \\
\hline & Model 3 & $16.64(6.6)$ & 0.01 & $19.38(5.1)$ & 0.00 & $2.75(5.6)$ & 0.62 & $9.46(3.8)$ & 0.01 & $9.26(4.5)$ & 0.04 \\
\hline & Model 4 & $15.20(6.7)$ & 0.02 & $20.01(5.4)$ & 0.00 & $2.50(5.5)$ & 0.65 & $9.64(4.0)$ & 0.02 & $9.16(4.6)$ & 0.05 \\
\hline \multirow[t]{4}{*}{ LVEF (\%) } & Model 1 & $-2.04(1.1)$ & 0.07 & $-0.92(1.0)$ & 0.37 & $-0.58(1.0)$ & 0.55 & $-1.03(1.0)$ & 0.30 & $-1.67(1.0)$ & 0.09 \\
\hline & Model 2 & $-1.37(1.1)$ & 0.22 & $-0.40(1.0)$ & 0.68 & $-0.12(0.9)$ & 0.90 & $-0.56(1.0)$ & 0.55 & $-1.29(1.0)$ & 0.18 \\
\hline & Model 3 & $-1.32(1.1)$ & 0.24 & $-0.35(1.0)$ & 0.73 & $-0.16(0.9)$ & 0.87 & $-0.57(1.0)$ & 0.55 & $-1.23(1.0)$ & 0.21 \\
\hline & Model 4 & $-0.76(1.1)$ & 0.52 & $-0.16(1.0)$ & 0.88 & $-0.19(0.9)$ & 0.85 & $-0.41(1.0)$ & 0.69 & $-1.20(1.0)$ & 0.25 \\
\hline
\end{tabular}

${ }^{*} \beta$ Estimate is the estimated change in outcome variable per unit change in predictor if all other variables remain constant.

EDV, end-diastolic volume; ESV, end-systolic volume; LAVI, left atrial volume index; LVEF, left ventricular ejection fraction; LVM, left ventricular mass.

Model 1: Age

Model 2: Age, BMI, SBP, diabetes or pre-diabetes and anti-hypertension medication use

Model 3: Smoking status, total and $\mathrm{HDL}$ cholesterol in addition to variables in model 2

Model 4: Education and household income in addition to variables in model 3

The methods of evaluation and grading of DD are also diverse across prior studies, so methodology may account for some of this difference in prevalence of DD. We have recently published data characterising the burden of systolic dysfunction and DD from the entire ECHO-SOL cohort in greater detail and with further comparison to prior studies. ${ }^{30}$ In our sensitivity analysis we also assessed DD using the newer 2016 guidelines and the overall prevalence of DD was lower. However, these criteria were not used in the main analysis as these guidelines themselves are a work in progress, based on expert consensus, which have not been validated and we wished to place our work in context with prior studies.

\section{Underlying mechanisms}

The underlying mechanisms for the cardiovascular physiological adaptations during pregnancy that persist after pregnancy are not well understood. Both oestrogen and progesterone, in various concentrations, have been known to induce hypertrophy and may be partially responsible for the physiological hypertrophy of pregnancy. ${ }^{39}$ Relaxin plays a vital role in vasodilation and angiogenesis as well as protecting the LV from fibrosis and inflammation through downstream effects of nitrous oxide, vascular endothelial growth factor, endothelins, matrix metalloproteinases, tumour necrosis factor $\alpha$ and transforming growth factor $\beta .^{41} \mathrm{~A}$ delicate balance between matrix metalloproteinases and tissue inhibitors of metalloproteinases regulate extracellular matrix changes which both facilitate relaxation of tissues for the process of childbirth and protect the extracellular matrix of the heart from fibrosis during these adaptations. ${ }^{42} 43$ However, little is known about the long-term effects of these adaptations when they occur repeatedly. Prior studies have observed poor cardiac performance, increased cardiac volumes and hypertrophy in female breeder rats compared with virgin female rats. ${ }^{44}$ We propose that these adaptations across successive pregnancies may not revert to prepregnancy states, thus having long-lasting effects such as extra-cellular matrix (ECM) remodelling, alteration of collagen, apoptosis, replacement fibrosis and hypertrophy leading to possible LV stiffening, loss of contractile function and DD. Although our analysis showed an independent effect of greater parity with abnormal cardiac structure and DD, these 

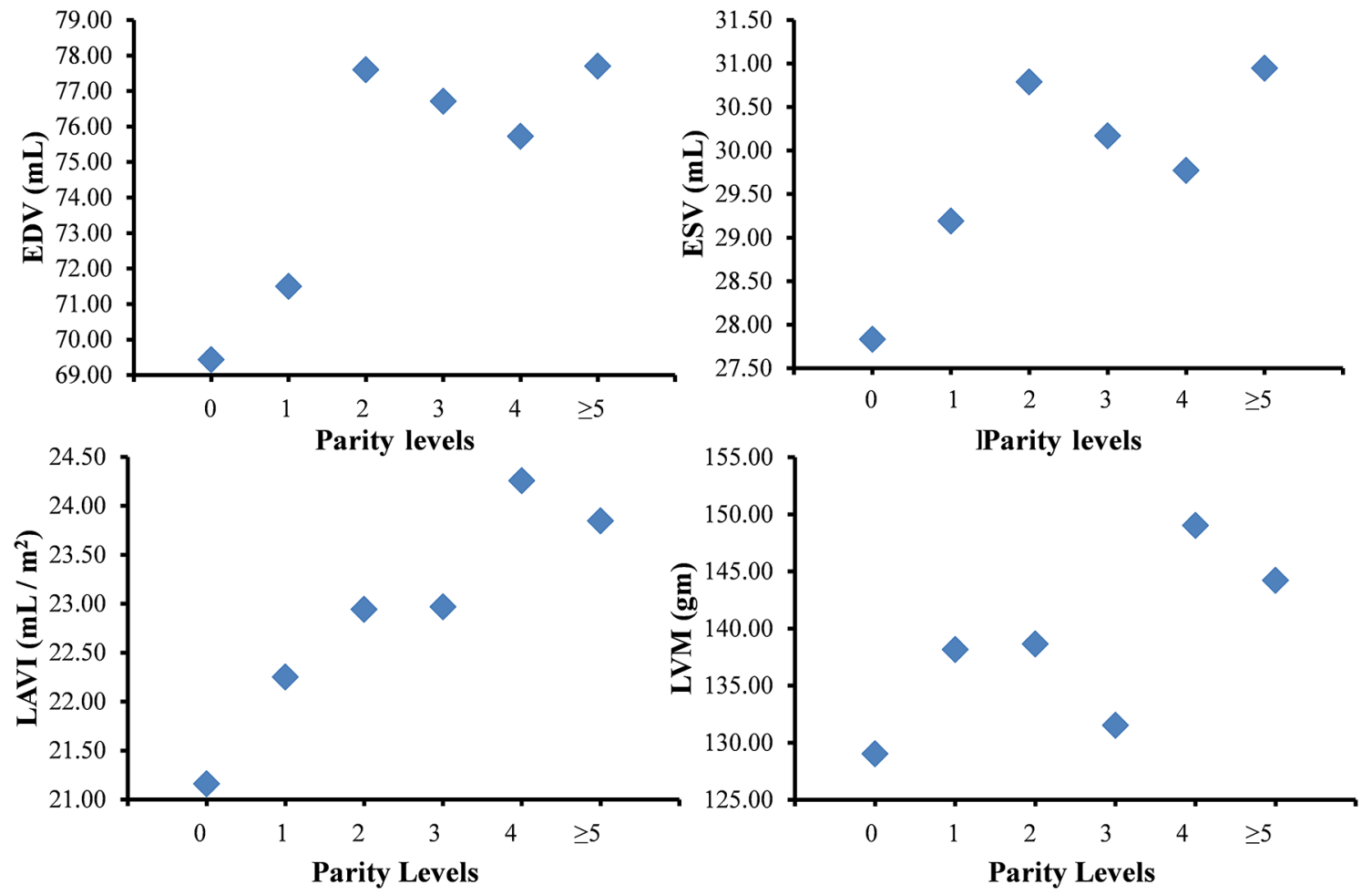

Figure 2 Least square means for changes in echocardiographic variables with parity. Least square means for various echocardiographic parameters and their association with increasing levels of parity in fully adjusted models for age, body mass index, diabetes or prediabetes, systolic blood pressure, antihypertensive medications, smoking, total cholesterol and highdensity lipoprotein cholesterol, education and income. EDV, end-diastolic volume; ESV, end-systolic volume; LAVI, left atrial volume index; LVM, left ventricular mass.

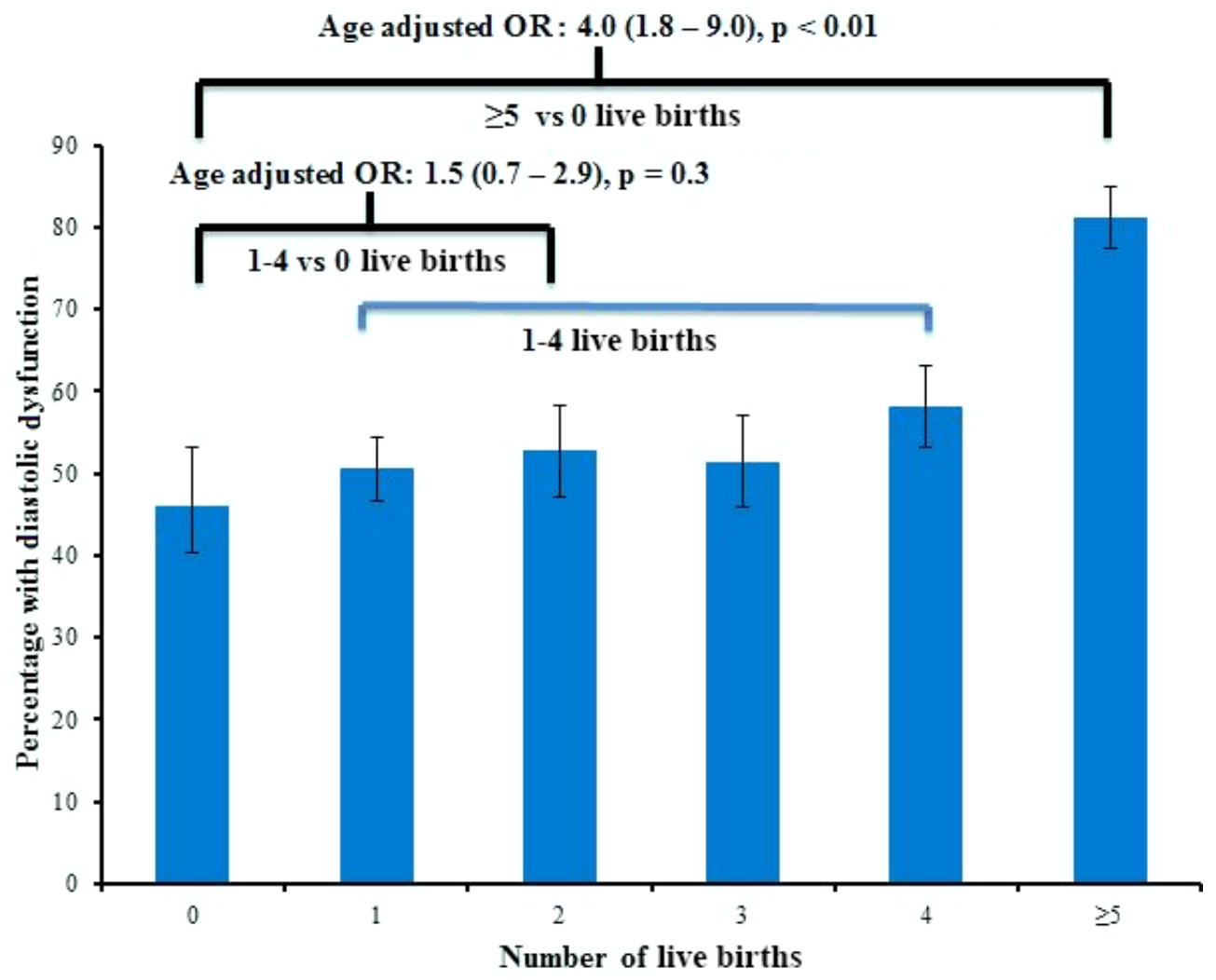

Figure 3 Prevalence of any-grade diastolic dysfunction by levels of parity. 


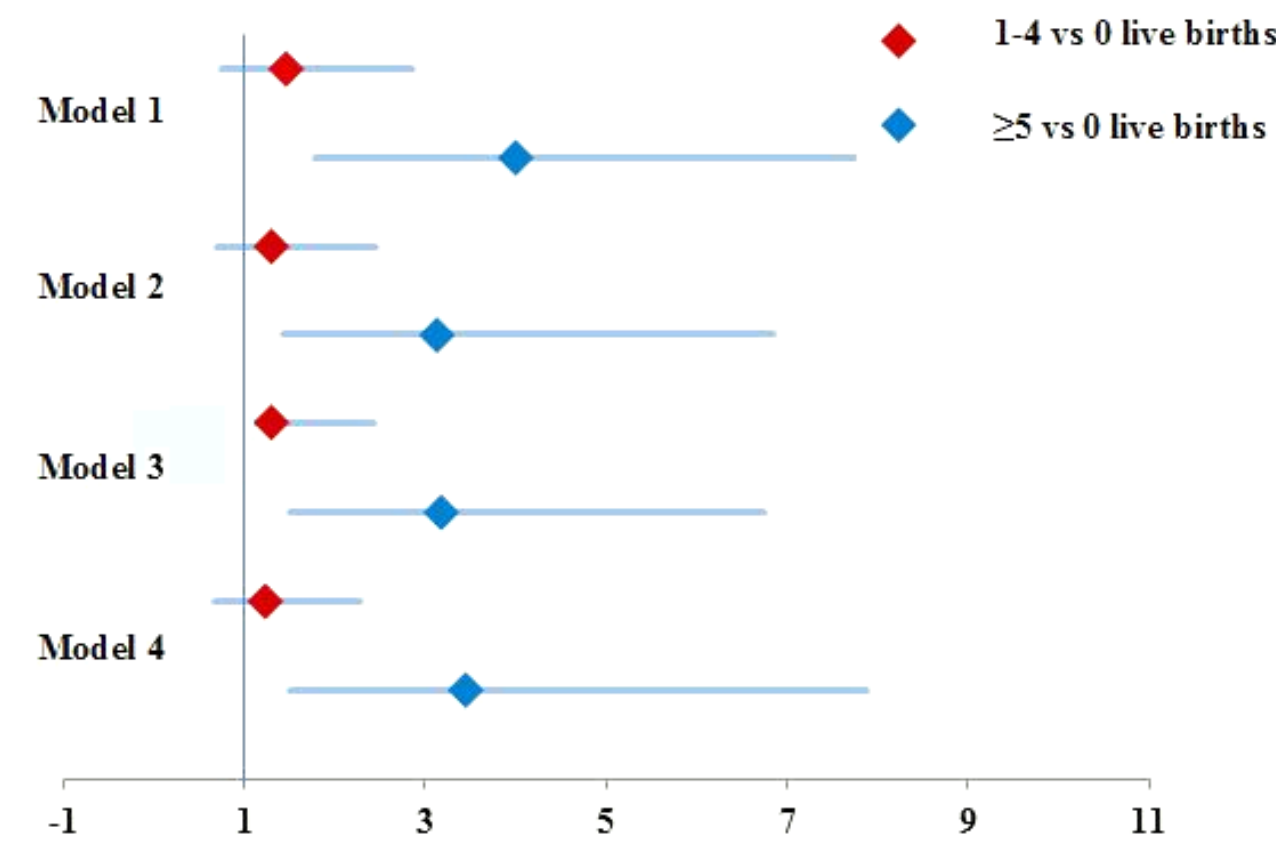

Figure 4 ORs for logistic regression models of association between parity and any-grade diastolic dysfunction Model 1: Adjusted for age; Model 2: Adjusted for age, body mass index, systolic blood pressure, diabetes or prediabetes and antihypertension medication use; Model 3: Adjusted for smoking status, total cholesterol and high-density lipoprotein cholesterol in addition to variables in model 2; Model 4: Adjusted for education and household income in addition to variables in model 3.

relations could be greatly compounded by multiple risk factors for CVD and possible mediators such as adiposity redistribution and hypertension during pregnancy. Increased adipose significantly impairs diastolic function, ${ }^{45}$ and since pregnancy usually adds about $5-10$ pounds of body fat long term, there may a strong role for adiposity to explain our findings.

\section{Implications of our findings}

Our study demonstrates the possibility of higher parity as a novel risk factor for abnormal cardiac structure and function. Several studies have shown the predictive value of echocardiographic DD in the development of clinical $\mathrm{HFpEF}$ and its association with increased morbidity and mortality in this population. ${ }^{46}$ Given the epidemic of HFpEF in our ageing population, particularly in women, identifying those at high risk would be of prime importance for early identification, treatment and prevention. It may be important for the cardiologist or general practitioner to inquire into the obstetric history of women in more detail, with concerns of high parity as a risk factor for adverse LV remodelling, DD and possibly future HFpEF. Furthermore, populations with women with very high parity, such as Hispanic/Latina women, ${ }^{48}{ }^{49}$ may need closer follow-up and screening.

It is interesting that previous studies indicate that there may be a quadratic or J-shaped relationship between parity and clinical outcomes, specifically mortality. ${ }^{50}$ Our results indicate a significant increase in LV volumes with two or more live births; increase in mass even with one live birth and DD with five or more live births; however the relationship was not entirely linear. For example,
EDV and ESV increased after two live births compared with nulliparous women with only minor increases thereafter. Further research is needed to further clarify if there is a cut-off in the number of live births after which women are at the highest risk for adverse cardiovascular outcomes and to clarify if having a low number of live births provides potential protective effects. Earlier studies do reveal a lower mortality in women with a low number of live births compared with nulliparous women and a higher mortality in grand-multiparous women. However, this relationship may have been due to a higher number of accidental deaths in nulliparous women and increased CVD-related deaths in grand-multiparous women. ${ }^{50}$ It is possible that while the initial adaptations to pregnancy may have some overall benefits, recurrent adaptations across successive pregnancies leave insufficient time for complete return to baseline, thus preventing the cardiovascular system from being able to fully compensate.

\section{Limitations}

While our study has the benefit of a well-characterised population with high parity, our findings need to be verified in other populations. Our study was unable to account for the time interval between pregnancies, which could have a significant effect on the observed associations. However, this is a very difficult predictor to evaluate, especially since the intervals may vary considerably. Furthermore, we did not have information on the times since the last pregnancy though some of these factors may be partially accounted for by age and the total number of pregnancies. We could not confirm the presence of multiple births or the effects of abortions 
or miscarriages and there may be some misclassification of the predictor though we believe the effect would be minimal. We could not adjust for potential confounders such as history of pre-eclampsia, gestational diabetes and gestational hypertension, though some of these effects may be accounted for by the presence of prediabetes or higher SBP, which were included in the models. However despite adjustment for various risk factors for DD such as age, weight, hypertension and diabetes, residual confounding is a concern. We also lacked information on the presence of peripartum cardiomyopathy; however, relative to the magnitude of the effects we found, the prevalence of peripartum cardiomyopathy is not likely to be significant. We also lacked information on the psychosocial effects of raising children (childrearing) which could be a confounder given the association of stress with CVD.

\section{CONCLUSIONS}

High parity is associated with greater abnormalities of cardiac structure and function and in particular a significantly higher prevalence of DD among Hispanic/Latina women suggesting that parity is a novel risk factor for abnormalities of cardiac structure and function. Further studies are needed to determine the underlying pathophysiological mechanisms for these apparent deleterious consequences of higher parity on the LV, to determine whether these changes translate into HF outcomes, particularly HFpEF, and lastly to determine if there are any potential preventive strategies.

\section{Acknowledgements The authors thank the investigators (www.cscc.unc. edu/hchs/), the staff and the participants of HCHS-SOL and ECHO-SOL for their dedication and commitment to the success of this study.}

Funding The HCHS/SOL was carried out as a collaborative study supported by contracts from the National Heart, Lung, and Blood Institute (NHLBI) to the University of North Carolina (N01- HC65233), University of Miami (N01-HC65234), Albert Einstein College of Medicine (N01-HC65235), Northwestern University (N01HC65236) and San Diego State University (N01-HC65237). The following Institutes/ Centres/Offices contribute to the HCHS/SOL through a transfer of funds to the NHLBI: National Institute on Minority Health and Health Disparities, National Institute on Deafness and Other Communication Disorders, National Institute of Dental and Craniofacial Research, National Institute of Diabetes and Digestive and Kidney Diseases, National Institute of Neurological Disorders and Stroke, NIH InstitutionOffice of Dietary Supplements. ECHO-SOL was supported by a grant from the NHLBI (R01 HL104199, Epidemiologic Determinants of Cardiac Structure and Function among Hispanics: CJR, Principal Investigator).

Competing interests None declared.

Provenance and peer review Not commissioned; internally peer reviewed.

Data sharing statement All HCHS-SOL data will be included in a limited access database that will be available to the larger scientific public as part of the main HCHS-SOL study database for public use. It will be encouraged that all use of data and publications from ancillary studies invite the participation of the ancillary study $\mathrm{PI}$ and co-investigators. For more information on HCHS/SOL related limited access datasets see the URL: http://www.cscc.unc.edu/hchs/.

Open Access This is an Open Access article distributed in accordance with the Creative Commons Attribution Non Commercial (CC BY-NC 4.0) license, which permits others to distribute, remix, adapt, build upon this work non-commercially, and license their derivative works on different terms, provided the original work is properly cited and the use is non-commercial. See: http://creativecommons.org/ licenses/by-nc/4.0/ (c) Article author(s) (or their employer(s) unless otherwise stated in the text of the article) 2017. All rights reserved. No commercial use is permitted unless otherwise expressly granted.

\section{REFERENCES}

1. Owan TE, Hodge DO, Herges RM, et al. Trends in prevalence and outcome of heart failure with preserved ejection fraction. $N$ Engl J Med 2006;355:251-9.

2. Lee DS, Gona P, Vasan RS, et al. Relation of disease pathogenesis and risk factors to heart failure with preserved or reduced ejection fraction: insights from the framingham heart study of the national heart, lung, and blood institute. Circulation 2009;119:3070-7.

3. Borlaug BA, Redfield MM. Diastolic and systolic heart failure are distinct phenotypes within the heart failure spectrum. Circulation 2011;123:2006-14. discussion 14.

4. Brouwers FP, de Boer RA, van der Harst P, et al. Incidence and epidemiology of new onset heart failure with preserved vs. reduced ejection fraction in a community-based cohort: 11-year follow-up of PREVEND. Eur Heart J 2013;34:1424-31.

5. Kametas NA, McAuliffe F, Hancock J, et al. Maternal left ventricular mass and diastolic function during pregnancy. Ultrasound Obstet Gynecol 2001;18:460-6.

6. Poppas A, Shroff SG, Korcarz CE, et al. Serial assessment of the cardiovascular system in normal pregnancy. role of arterial compliance and pulsatile arterial load. Circulation 1997;95:2407-15.

7. Melchiorre K, Sharma R, Thilaganathan B, et al. Cardiac structure and function in normal pregnancy. Curr Opin Obstet Gynecol 2012;24:413-21.

8. Hunter S, Robson SC. Adaptation of the maternal heart in pregnancy. Heart 1992;68:540-3.

9. Katz R, Karliner JS, Resnik R. Effects of a natural volume overload state (pregnancy) on left ventricular performance in normal human subjects. Circulation 1978;58:434-41.

10. Ness RB, Harris T, Cobb J, et al. Number of pregnancies and the subsequent risk of cardiovascular disease. N Engl J Med 1993;328:1528-33.

11. Beral V. Long term effects of childbearing on health. $J$ Epidemiol Community Health 1985;39:343-6.

12. Dekker JM, Schouten EG. Number of pregnancies and risk of cardiovascular disease. N Engl J Med 1993;329:1893-4. author reply 94-5.

13. Green A, Beral V, Moser K. Mortality in women in relation to their childbearing history. Bmj 1988;297:391-5.

14. Catov JM, Newman AB, Sutton-Tyrrell K, et al. Parity and cardiovascular disease risk among older women: how do pregnancy complications mediate the association? Ann Epidemiol 2008;18:873-9.

15. Vladutiu CJ, Siega-Riz AM, Sotres-Alvarez D, et al. Parity and components of the metabolic syndrome among US hispanic/Latina women: results from the hispanic community health Study/Study of latinos. Circ Cardiovasc Qual Outcomes 2016;9(2 Suppl 1):S62-9.

16. Schannwell CM, Schoebel FC, Zimmermann T, et al. Left ventricular diastolic function in normal pregnancy. A prospective study using M-mode echocardiography and Doppler echocardiography]. Dtsch Med Wochenschr 2000;125:1069-73.

17. Clapp JF, Capeless E. Cardiovascular function before, during, and after the first and subsequent pregnancies. Am J Cardiol 1997;80:1469-73.

18. Parikh NI, Lloyd-Jones DM, Ning H, et al. Association of number of live births with left ventricular structure and function. The Multi-Ethnic Study of Atherosclerosis (MESA). Am Heart $J$ 2012;163:470-6.

19. Lavange LM, Kalsbeek WD, Sorlie PD, et al. Sample design and cohort selection in the hispanic community health Study/Study of latinos. Ann Epidemiol 2010;20:642-9.

20. Rodriguez CJ, Dharod A, Allison MA, et al. Rationale and Design of the Echocardiographic Study of Hispanics / Latinos (ECHO-SOL). Ethnicity and Disease 2015;25:180-6.

21. Rodriguez CJ, Allison MA, Shah SJ, et al. And design of the echocardiographic study of hispanics / Latinos (ECHO-SOL). Ethnicity \& Disease 2014.

22. Gilman G, Nelson TA, Hansen WH, et al. Diastolic function: a sonographer's approach to the essential echocardiographic measurements of left ventricular diastolic function. J Am Soc Echocardiogr 2007;20:199-209.

23. Lang RM, Bierig M, Devereux RB, et al. Chamber Quantification Writing GroupAmerican Society of Echocardiography's Guidelines and Standards CommitteeEuropean Association of Echocardiography. Recommendations for chamber quantification: a 
report from the american society of echocardiography's Guidelines and Standards Committee and the Chamber Quantification Writing Group, developed in conjunction with the European Association of Echocardiography, a branch of the European Society of Cardiology. $J$ Am Soc Echocardiogr 2005;18:1440-63.

24. Byrd BF, Finkbeiner W, Bouchard A, et al. Accuracy and reproducibility of clinically acquired two-dimensional echocardiographic mass measurements. Am Heart $J$ 1989;118:133-7.

25. Devereux RB, Alonso DR, Lutas EM, et al. Echocardiographic assessment of left ventricular hypertrophy: comparison to necropsy findings. Am J Cardiol 1986;57:450-8.

26. Lang RM, Badano LP, Mor-Avi V, et al. Recommendations for cardiac chamber quantification by echocardiography in adults: an update from the american society of echocardiography and the european association of cardiovascular imaging. J Am Soc Echocardiogr 2015;28:e14:1-39.

27. Nagueh SF, Appleton CP, Gillebert TC, et al. Recommendations for the evaluation of left ventricular diastolic function by echocardiography. Eur J Echocardiogr 2009;10:165-93.

28. Nagueh SF, Appleton CP, Gillebert TC, et al. Recommendations for the evaluation of left ventricular diastolic function by echocardiography. J Am Soc Echocardiogr 2009;22:107-33.

29. Redfield MM, Jacobsen SJ, Burnett JC, et al. Burden of systolic and diastolic ventricular dysfunction in the community: appreciating the scope of the heart failure epidemic. Jama 2003;289:194-202.

30. Mehta H, Armstrong A, Swett K, et al. Burden of systolic and diastolic left ventricular dysfunction among hispanics in the united states: insights from the echocardiographic study of latinos. Circulation Heart failure 2016;9:4.

31. Nagueh SF, Smiseth OA, Appleton CP, et al. Recommendations for the evaluation of left ventricular diastolic function by echocardiography: an update from the american society of echocardiography and the european association of Cardiovascular Imaging. J Am Soc Echocardiogr 2016;29:277-314.

32. Byrd BF, Finkbeiner W, Bouchard A, et al. Accuracy and reproducibility of clinically acquired two-dimensional echocardiographic mass measurements. Am Heart $J$ 1989;118:133-7.

33. Devereux RB, Alonso DR, Lutas EM, et al. Echocardiographic assessment of left ventricular hypertrophy: comparison to necropsy findings. Am J Cardiol 1986:57:450-8.

34. Daviglus ML, Talavera GA, Avilés-Santa ML, et al. Prevalence of major cardiovascular risk factors and cardiovascular diseases among hispanic/Latino individuals of diverse backgrounds in the united states. Jama 2012;308:1775-84.

35. Sorlie PD, Allison MA, Avilés-Santa ML, et al. Prevalence of hypertension, awareness, treatment, and control in the hispanic community health Study/Study of latinos. Am J Hypertens 2014:27:793-800.
36. Schneiderman N, Llabre M, Cowie CC, et al. Prevalence of diabetes among hispanics/Latinos from diverse backgrounds: the hispanic community health Study/Study of latinos (HCHS/SOL). Diabetes Care 2014;37:2233-9.

37. Kloch-Badelek M, Kuznetsova T, Sakiewicz W, et al. European Project On Genes in Hypertension (EPOGH) Investigators. Prevalence of left ventricular diastolic dysfunction in european populations based on cross-validated diagnostic thresholds. Cardiovasc Ultrasound 2012;10:10.

38. Kuznetsova T, Herbots L, López B, et al. Prevalence of left ventricular diastolic dysfunction in a general population. Circ Heart Fail 2009;2:105-12.

39. Chung E, Yeung F, Leinwand LA. Akt and MAPK signaling mediate pregnancy-induced cardiac adaptation. J Appl Physiol 2012;112:1564-75

40. Kilić A, Javadov S, Karmazyn M. Estrogen exerts concentrationdependent pro-and anti-hypertrophic effects on adult cultured ventricular myocytes. role of NHE-1 in estrogen-induced hypertrophy. J Mol Cell Cardiol 2009;46:360-9.

41. Teichman SL, Unemori E, Teerlink JR, et al. Relaxin: review of biology and potential role in treating heart failure. Curr Heart Fail Rep 2010;7:75-82.

42. Chung E, Leinwand LA. Pregnancy as a cardiac stress model. Cardiovasc Res 2014;101:561-70.

43. Umar S, Nadadur R, lorga A, et al. Cardiac structural and hemodynamic changes associated with physiological heart hypertrophy of pregnancy are reversed postpartum. J Appl Physiol 2012;113:1253-9.

44. Shreiner DP, Weisfeldt ML, Shock NW. Effects of age, sex, and breeding status on the rat heart. Am J Physiol 1969;217:176-80.

45. Kitzman DW, Shah SJ. The HFpEF obesity phenotype: the elephant in the room. J Am Coll Cardiol 2016;68:200-3.

46. Aurigemma GP, Gottdiener JS, Shemanski L, et al. Predictive value of systolic and diastolic function for incident congestive heart failure in the elderly: the cardiovascular health study. J Am Coll Cardiol 2001;37:1042-8.

47. Zile MR, Gottdiener JS, Hetzel SJ, et al. I-PRESERVE Investigators. Prevalence and significance of alterations in cardiac structure and function in patients with heart failure and a preserved ejection fraction. Circulation 2011;124:2491-501.

48. Radecki SE. A racial and ethnic comparison of family formation and contraceptive practices among low-income women. Public Health Rep 1991;106:494-502.

49. Aliyu MH, Salihu HM, Keith LG, et al. Trends in birth across highparity groups by race/ethnicity and maternal age. J Natl Med Assoc 2005;97:799-804.

50. Koski-Rahikkala $\mathrm{H}$, Pouta $\mathrm{A}$, Pietiläinen $\mathrm{K}$, et al. Does parity affect mortality among parous women? J Epidemiol Community Health 2006:60:968-73. 